\title{
Air-water: two phase flow behavior in a horizontal pipe using computational fluids dynamics (CFD)
}

\author{
F. Vásquez, M. Stanko, A. Vásquez, J. De Andrade \\ \& M. Asuaje \\ Laboratory of Mechanical Energy Conversion, Simón Bolivar University, \\ Venezuela
}

\begin{abstract}
Two-phase flow is an area of primary interest, particularly for the petroleum industry, where a fair amount of these kinds of flows can be found in the production of oil wells. This study refers to the evaluation of two-phase flow airwater through a horizontal pipe of $0.0508 \mathrm{~m}$ (2in) diameter and $1.016 \mathrm{~m}$ (40in) length. A 3D CFD approach was used for reproducing the behavior of the dispersed bubble, stratified smooth and slug flow in a horizontal pipe. The pressure drop and liquid holdup associated with it were estimated. Data of the superficial velocities of the liquid and gas were taken from the literature; and then many CFD simulations were carried out using different multiphase flow models. The results were validated with the available experimental data from the literature. For the case in which the liquid is the continuous phase and the gas behaves as the dispersed one, the results show that the bubble disperse flow behaves homogeneously. In these simulations, for a $0.01 \mathrm{~mm}$ gas bubble diameter the pressure drop presented a deviation of $6.12 \%$ over that reported in the literature. For the liquid holdup value a difference of $0.001 \%$ was obtained. An inhomogeneous approach with the free surface model reproduced satisfactorily the stratified smooth behavior. In addition, the influences of increasing water superficial velocity on the energy losses and the liquid holdup were obtained. However, for the studied numerical domain, the slug flow pattern could not be reproduced. All these results above allowed us to establish the great applicability of CFD modeling in the problem resolution of two-phase flow in a horizontal pipe.

Keywords: two-phase flow, CFD, flow patterns, liquid holdup, pressure drop.
\end{abstract}




\section{Introduction}

In the petroleum industry, the two-phase flow characterization has an essential importance due to its presence during the production and transportation of crude oil and natural gas. The gas and liquid phases are distributed in the pipe in a variety of flow configuration, called flow patterns. The flow pattern prediction is a major problem in two-phase flow analysis. Indeed, main variables like: pressure drop, liquid holdup, heat and mass transfer coefficients are strongly dependent on the existing flow pattern. These variables have to be predicted in order to reduce the main potentially hazardous problems that such parameters could cause.

The explanation above is why many researchers study the two-phase flow behavior in horizontal and vertical pipes, trying to predict accurately the flow pattern. In that sense, Aziz and Petalas [1] proposed a mechanistic model applicable to all pipe geometries and fluid properties. Thus, new empirical correlations were proposed for: liquid/wall and liquid/gas interfacial friction in stratified flow, the liquid fraction entrained and the interfacial friction in annular-mist flow, and the distribution coefficient used in the determination of holdup in intermittent flow. The model application has shown the ability to predict reasonably accurate pressure drops and holdup under different operating conditions. Wongwises et al. [2] presented new correlations to predict the liquid holdup with horizontal concurrent stratified flow in a circular pipe. Also, they demonstrated that the liquid holdup can be predicted by using Taitel and Dukler momentum balance between both phases. Recently, Shoham [3] presented an approach for flow patterns prediction. The mathematical mechanistic model was based on the physical phenomena of the two-phase behavior analysis. The model allows its application to diverse operational conditions, showing results with significant confidence.

With nowadays computer performances and dedicated codes, numerical simulations of tridimensional two-phase flow calculation in a horizontal pipe are proposed with reasonable precision, providing a close look at the flow development through the pipe. In this new direction of two-phase flow understanding, the present study assesses the two-phase air-water flow in a horizontal pipe by means of 3D CFD flow simulations. The study mainly focus on predicting the behavior of the flow patterns, the pressure drop in the flow line, as well as on predicting the liquid holdup changes throughout the pipe. The specific objectives of the study are to:

- Reproduce the flow patterns behavior, for air-water mixture in a horizontal pipe by means of CFD techniques.

- Determine the pressure drop associated to the flow pattern under study.

- Determine the liquid holdup associated to each flow pattern through the pipe.

- Validate with literature data, the results obtained by the simulations.

- Establish the applicability of CFD techniques in the calculation of two-phase flow in a horizontal pipe. 


\section{Verification of flow patterns existence}

Based on the Taitel and Dukler [4] flow patterns map, the studied flow patterns were selected. Due to the complexity of the flow patterns identification by means of visual observations, it was important to verify the flow patterns predicted by Taitel and Dukler for all the selected values of superficial velocities of water and air, against some correlations. This verification was carried out using the specific correlations presented in table 1, which are widely used in this research area [5].

Table 1: $\quad$ Test case values of water and air superficial velocity, for each flow pattern considered.

\begin{tabular}{cccc}
\hline $\begin{array}{c}\text { Taitel and } \\
\text { Dukler flow } \\
\text { pattern }\end{array}$ & $\begin{array}{c}\mathbf{V}_{\mathbf{S L}} \\
{[\mathbf{m} / \mathbf{s}]}\end{array}$ & $\begin{array}{c}\mathbf{V}_{\mathbf{S G}} \\
{[\mathbf{m} / \mathbf{s}]}\end{array}$ & $\begin{array}{c}\text { Verification } \\
\text { correlations }\end{array}$ \\
\hline & 8.429 & 0.100 & \\
Dispersed bubble & 6.629 & 0.100 & Beggs and Brill \\
& 8.408 & 1.000 & \\
\hline & 6.881 & 1.000 & Guzhov \\
Stratified & 0.100 & 0.100 & \\
& 0.062 & 0.100 & Guzhov \\
& 0.010 & 0.100 & \\
\hline Slug & 0.100 & 1.000 & \\
& 0.062 & 1.000 & \\
& 0.010 & 1.000 & 10 \\
\end{tabular}

For dispersed bubble the Beggs and Brill correlation [6] was used. On the other hand, to verify the presence of the flows stratified smooth and slug the correlation of Guzhov was used [3].

\section{Numerical approach}

Numerical simulations were carried out on a horizontal pipe of L/D coefficient equal to twenty (20). Furthermore, as in any CFD problem, a sensibility analysis was performed to guarantee the independence of the results with respect to the numerical grid. For the calculations, unstructured tetrahedral cells with inflated layers were created. The total number of elements in the final grid was 644,620. The simulations were performed using the software package ANSYS CFX v.11. To reproduce all the flow patterns above mentioned, an air-water two-phase mixture at $25^{\circ} \mathrm{C}$ and an Eulerian approach were used. The turbulence and the two-phase flow models were selected according to the three studied flow patterns 
as it is shown in table 2. For dispersed bubble flow several gas bubble diameters were studied, among them are: 1, 0.1, 0.01 and $0.001 \mathrm{~mm}$.

Table 2: $\quad$ Specification of numerical models used for each studied flow pattern.

\begin{tabular}{cccc}
\hline Flow pattern & $\begin{array}{c}\text { Two-phase flow } \\
\text { model }\end{array}$ & $\begin{array}{c}\text { Turbelence } \\
\text { model }\end{array}$ & $\begin{array}{c}\text { Temporal } \\
\text { approach }\end{array}$ \\
\hline Dispersed bubble & $\begin{array}{c}\text { Homogeneous: } \\
\text { mixture and } \\
\text { particle }\end{array}$ & K-épsilon & Steady state \\
\hline Stratified & $\begin{array}{c}\text { Inhomogeneous: } \\
\text { mixture and } \\
\text { particle }\end{array}$ & $\begin{array}{c}\text { Shear Stress } \\
\text { Transport }\end{array}$ & Steady state \\
\hline Slug & $\begin{array}{c}\text { Inhomogeneous: } \\
\text { free surface and } \\
\text { particle }\end{array}$ & K-épsilon & $\begin{array}{c}\text { Unsteady \& } \\
\text { steady state }\end{array}$ \\
\hline
\end{tabular}

The equations describing the fluid flow through a specific domain need to be numerically closed stipulating the so-called boundary conditions. At the inlet of the domain, a total pressure condition and the volumetric fractions of the air and water were set. This condition is the most accurate due to the inflow energy is defined and the software is allowed to obtain gradients in velocity and pressure. At the outlet the mass flow was specified. Each flow rate of interest corresponds to a different superficial velocity. The viscous fluxes are computed with a "high resolution" scheme, which means that in regions with low variable gradients, a second order scheme is used. In areas where the gradients change sharply, a first order upwind scheme is used to maintain robustness. Furthermore, root mean squared convergence criteria with an average residual target of $1 \times 10^{-4}$ in mass, momentum and turbulence $(\mathrm{k}-\varepsilon)$ equations was used.

In order to validate the results obtained by CFD calculation, the pressure drop and holdup were compared with values calculated from experimental data in the literature. These values were estimated by using the Wallis homogeneous flow model [7] for dispersed bubble, Lockhart and Martinelli separated flow model [8] for stratified smooth and finally the Dukler and Hubbard model [9] for slug.

\section{Results and discussion}

All flow patterns existence was verified using the mentioned correlations criteria. The conditions of the used correlations for verifying the flow patterns existence were satisfied in all the studied points of the disperse bubble, stratified and slug flow. Afterward, in this section the fluid dynamic behavior of the flow patterns in the horizontal pipe is addressed for the operating conditions in Table 1. First, the flow patterns behavior are qualitatively and quantitatively studied by means of water volume fraction contours and superficial velocity calculations. Next, pressure drop and holdup are assessed though the pipeline. Finally, the numerical calculations are compared with experimental data from literature. 


\subsection{Flow patterns behavior}

\subsubsection{Dispersed bubble flow}

A good numerical convergence and representation of the fluid dynamic associated with dispersed bubble flow was reached by using the homogenous approach with the particle model (particles diameter: $0.01 \mathrm{~mm}$ ). This flow pattern could be easily identified as it is shown in the fig. 1 . The water volume fraction contour shows the dispersed gas phase as small bubbles into the continuous liquid phase.

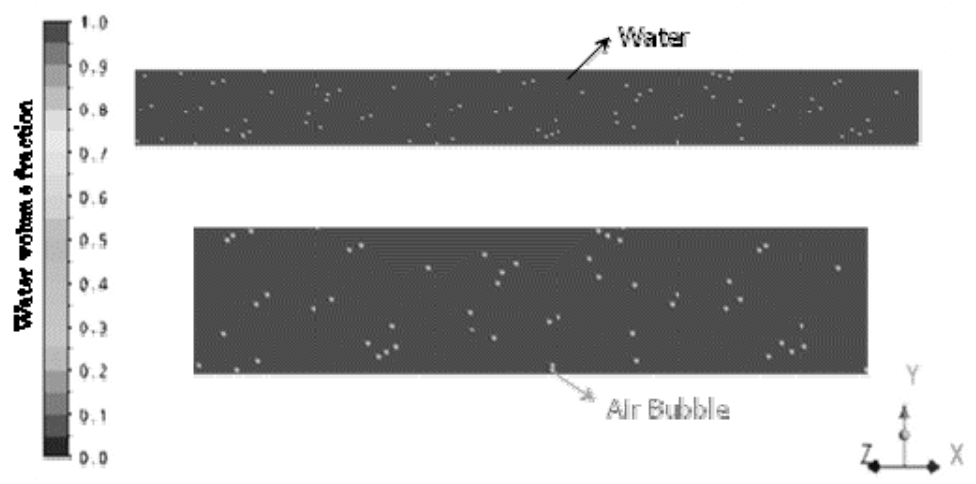

Figure 1: Dispersed bubble flow pattern throughout the pipe.

\subsubsection{Stratified flow}

This flow pattern was successfully reproduced with an inhomogeneous multiphase modeling, using the free surface model. In fig. 2, the liquid and gas velocities were plotted through the pipe. The figure presents a mixed flow at the inlet, and due to the buoyancy effect the phases are separated flowing through the pipe. The water flows at the bottom of the pipe and the air on the top. This stratified smooth flow takes place under relatively low flows of gas and liquid.

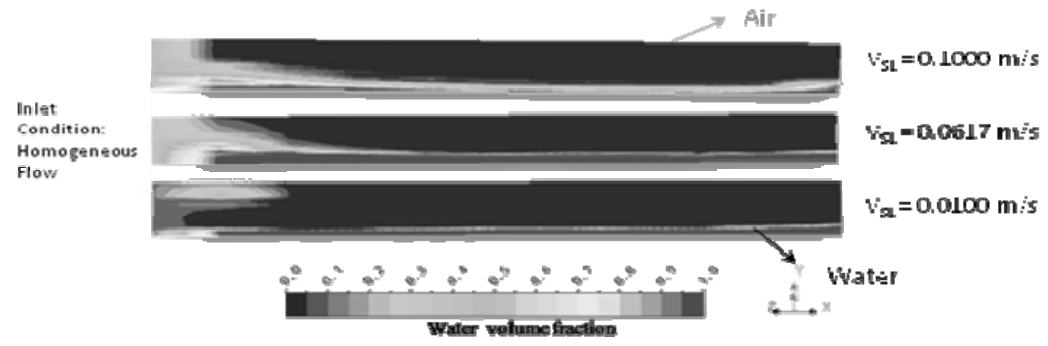

Figure 2: $\quad$ Stratified smooth flow pattern throughout the pipe. 
Figure 3 depicts the liquid and gas velocities. It is possible to see that the gas phase moves faster than the liquid one.

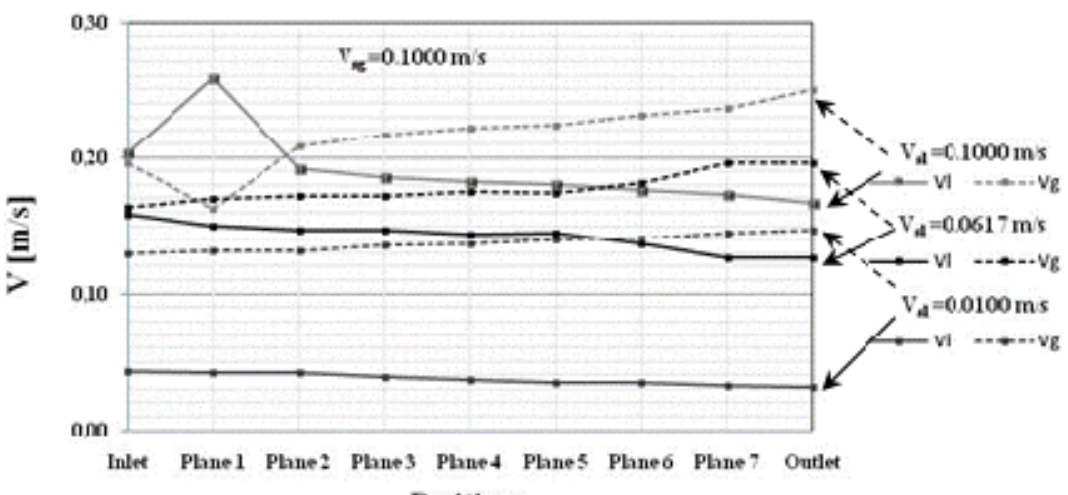

Positions

Figure 3: Velocities of the phases in stratified smooth flow pattern for $\mathrm{V}_{\mathrm{SG}}=0.10 \mathrm{~m} / \mathrm{s}$.

\subsubsection{Slug flow}

With regard to the slug flow pattern, in spite of the fact that a sinusoidal perturbation in the free surface model was set and a good numerical convergence was reached during the calculation, the slug flow configuration could not be reproduced. Even though the beginning of the flow stratification was observed, the slug flow was not completed; this could be attributed to the short pipe length which might not have been long enough to establish the stratification of the flow and to the slug development. These observations are in accordance with that Vallée et al. [10] reported with regard to the pipe length needed for slug generation, which can have an important influence on the evolution of the flow regime along the pipe.

\subsection{Pressure drop and holdup}

\subsubsection{Dispersed bubble flow}

In the figures 4 and 5 are shown the comparisons between obtained pressure drop for a $\mathrm{V}_{\mathrm{SG}}=0.1 \mathrm{~m} / \mathrm{s}$ and obtained pressure drop for a $\mathrm{V}_{\mathrm{SG}}=1 \mathrm{~m} / \mathrm{s}$, for several gas bubble diameter. As it was expected, the total pressure drop increases with increasing the superficial velocities of the liquid and gas phases. This is consistent with the fact of modeling the disperse bubble flow as a homogeneous flow with average physical properties. The relation between such parameters is directly proportional, so the greater the mixture velocity, the greater is the pressure obtained [3].

In addition, Figures 6 and 7 confirm the homogeneous flow condition in view of the fact that non slippage is observed between the liquid and the gas 


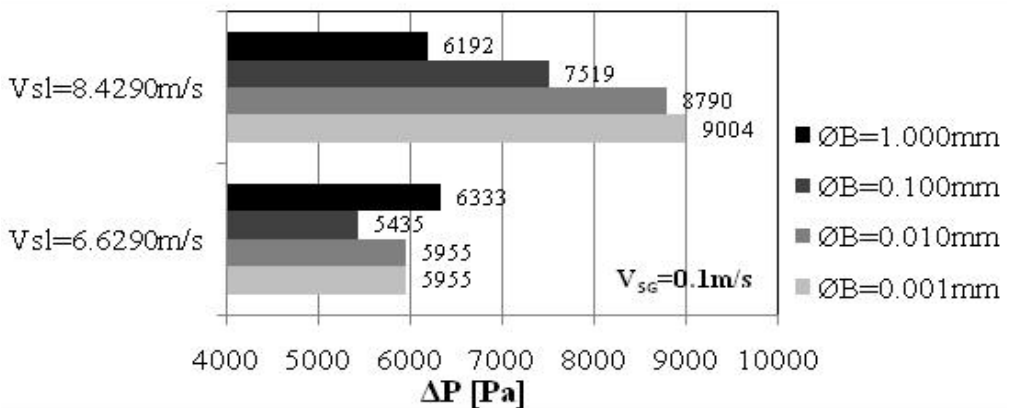

Figure 4: Total pressure drop in dispersed bubble for several bubble diameters and $\mathrm{V}_{\mathrm{SG}}=0.10 \mathrm{~m} / \mathrm{s}$.

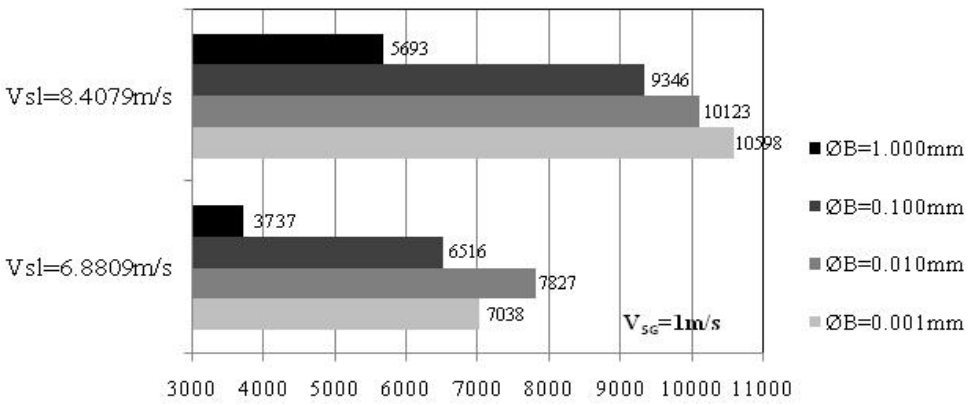

$\Delta \mathbf{P}[\mathbf{P a}]$

Figure 5: Total pressure drop in dispersed bubble for several bubble diameters and $\mathrm{V}_{\mathrm{SG}}=1 \mathrm{~m} / \mathrm{s}$.

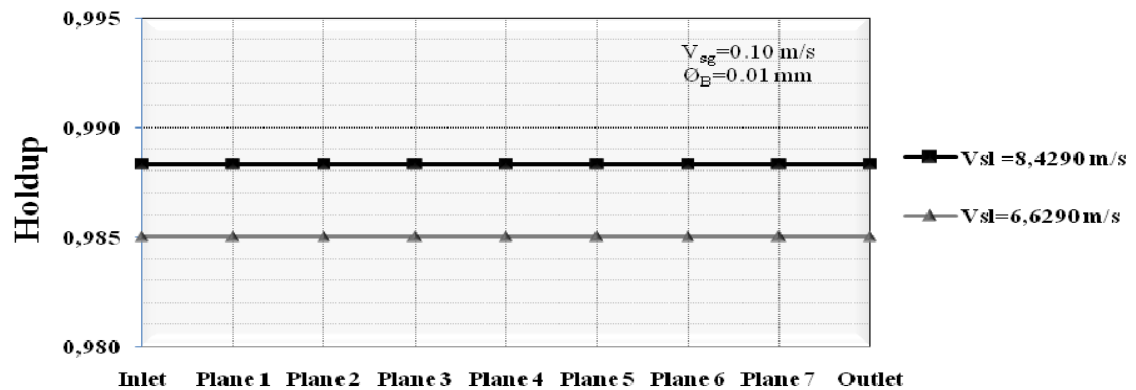

Positions

Figure 6: Holdup in dispersed bubble through the pipe for $\mathrm{V}_{\mathrm{SG}}=0.10 \mathrm{~m} / \mathrm{s}$. 


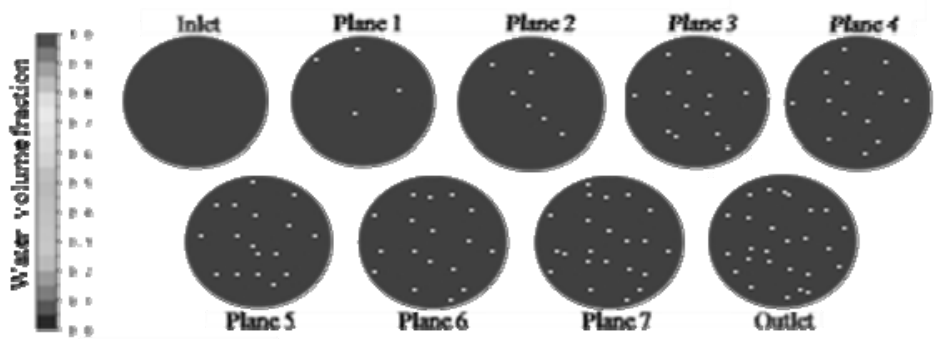

Figure 7: Holdup throughout each plane of the pipe for $\mathrm{V}_{\mathrm{SG}}=0.10 \mathrm{~m} / \mathrm{s}$ and $\mathrm{V}_{\mathrm{SL}}=8.42 \mathrm{~m} / \mathrm{s}$.

velocities. The gas bubbles are carried out by the liquid phase at the same velocity resulting in zero slippage. As it was expected, the holdup for each established superficial velocity remains constant.

\subsubsection{Stratified smooth flow}

The total pressure drop for a stratified flow is shown in the fig. 8. The increase of the superficial velocities of the phases leads to a significant pressure drop in the pipe; this occurs as a fluid dynamic response to the decrease of the total pressure at the outlet caused by the velocity increase, meaning greater pressure losses throughout the pipeline.

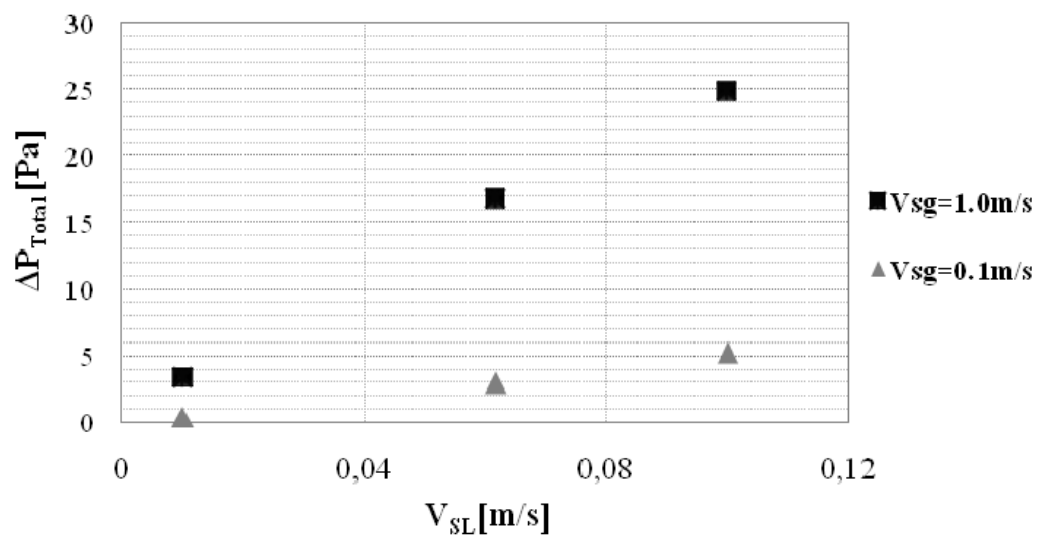

Figure 8: $\quad$ Total pressure drop in stratified smooth through the pipeline.

Fig. 9 shows the holdup along the pipe for several water superficial velocity values, keeping the air superficial velocity constant at $0.1 \mathrm{~m} / \mathrm{s}$. As it was expected, greater holdup was obtained as liquid superficial velocity was increased. This is consistent with the mass conservation consideration. As a result, an increase of the amount of the liquid results in an increase of the liquid area and a decrease of the gas area, simultaneously. This is reflected with a larger 
liquid accumulation in the bottom of the pipeline. Furthermore, fig. 10 shows how the slippage takes place between the two phases.

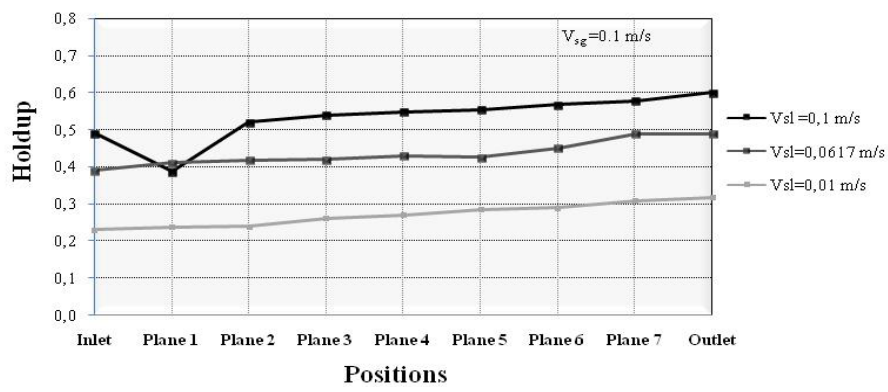

Figure 9: Holdup throughout each plane of the pipe for $\mathrm{V}_{\mathrm{SG}}=0.10 \mathrm{~m} / \mathrm{s}$ in stratified smooth.

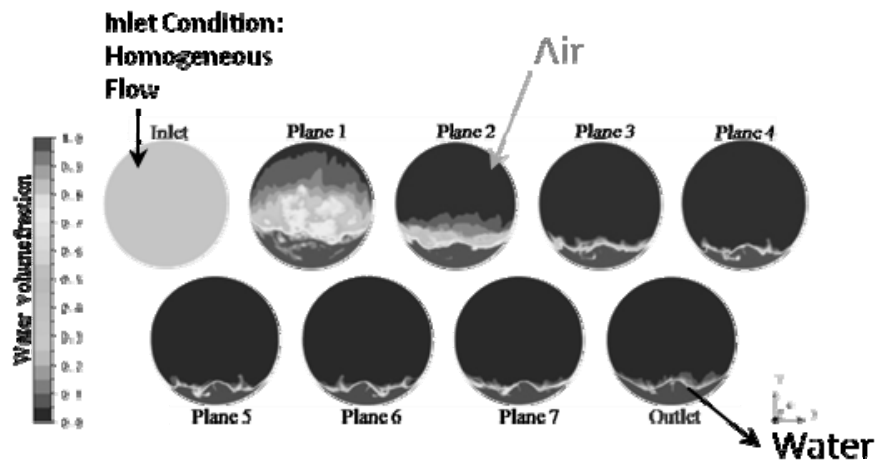

Figure 10: Holdup throughout each plane of the pipe for $\mathrm{V}_{\mathrm{SG}}=0.10 \mathrm{~m} / \mathrm{s}$ and $\mathrm{V}_{\mathrm{SL}}=0.0617 \mathrm{~m} / \mathrm{s}$, in stratified smooth.

\subsection{Comparison with experimental data from literature}

\subsubsection{Dispersed bubble flow}

With regard to the total pressure drop, in the figure 11it can be seen that for $0.01 \mathrm{~mm}$ and $0.001 \mathrm{~mm}$ bubble diameters, the obtained percentage deviations were smaller than $10 \%$. The particle size that reproduces better the dispersed bubble behavior is $0.01 \mathrm{~mm}$ (according to all the obtained results previously), which can be observed on the mentioned figure. This result was obtained for a $\mathrm{V}_{\mathrm{SG}}=1 \mathrm{~m} / \mathrm{s}$ as well. 


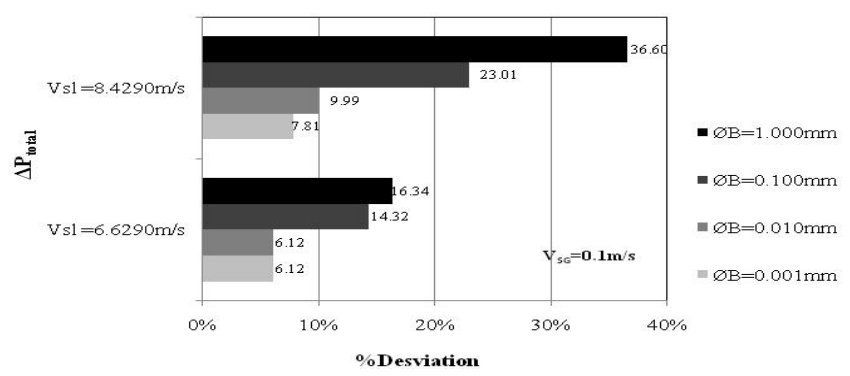

Figure 11: $\%$ Desviation of $\Delta \mathrm{P}_{\text {total }}$ in dispersed bubble for $\mathrm{V}_{\mathrm{SG}}=0.10 \mathrm{~m} / \mathrm{s}$.

In addition, with regard to holdup values, the error percentages are lower than $0.2 \%$ for all the particle sizes evaluated under different superficial velocity conditions (fig. 12). The outstanding results show consistency with the experimental data.

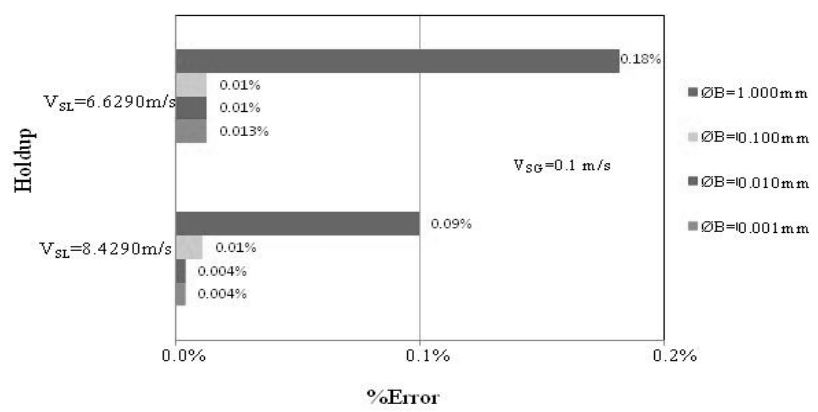

Figure 12: $\%$ Error of the holdup in dispersed bubble for $\mathrm{V}_{\mathrm{SG}}=0.10 \mathrm{~m} / \mathrm{s}$.

\subsubsection{Stratified smooth flow}

In fig. 13, a comparison of the results of pressure drop obtained by CFD calculation with those calculated according to the Lockhart and Martinelli model, it was found that error percentages fluctuated between $0.98 \%$ and $7.09 \%$, which shows a good accuracy.

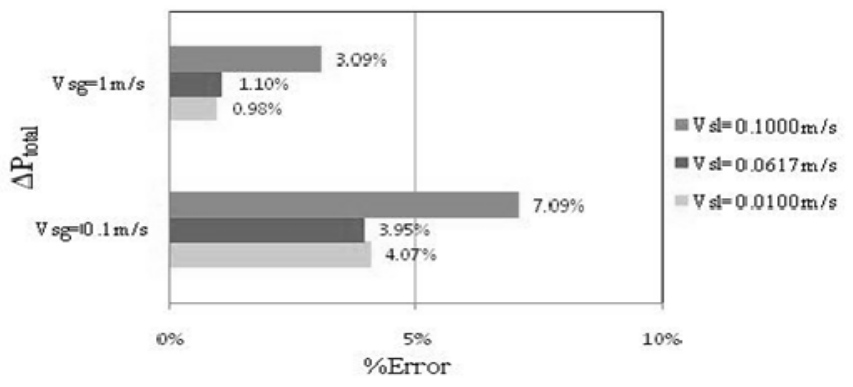

Figure 13: $\%$ Error of $\Delta \mathrm{P}_{\text {total }}$ in stratified smooth for different $\mathrm{V}_{\mathrm{sl}}$. 
Furthermore, figure 14 shows greater percentage of errors in the obtained holdup (between $2.55 \%$ and 20.24\%). Nevertheless, the results are considered acceptable.

In summary, the set of simulation models have been addressed to reproduce flow patterns for several operation conditions. The results could be considered satisfactory for each flow pattern, since the errors were lower than $20 \%$ in relation which those reported in literature [3]. Thus, the numerical approach is widely useful in problems resolution with two-phase flow in horizontal pipe.

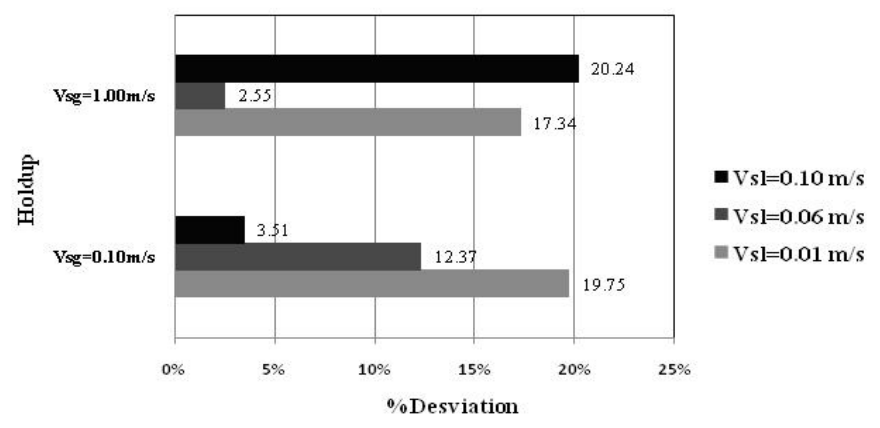

Figure 14: \%Error of holdup in stratified smooth for different $\mathrm{V}_{\mathrm{sl}}$.

\section{Conclusions}

1. Two-phase air water flow behavior was evaluated in a horizontal pipe, $\phi=2 \mathrm{in}$, by means of 3D CFD calculations.

2. Dispersed bubble flow pattern was reproduced accurately.

3. The dispersed bubble flow behaved homogenous. This result was obtained by using a homogenous modeling with the particle model, $0.01 \mathrm{~mm}$ bubble diameter. For this diameter, the greater the mixture velocity, the greater was the obtained total pressure drop. The liquid holdup is remained constant, for each assessed air superficial velocity.

4. The inhomogeneous approach with free surface model reproduced in an expected way the stratified flow. As the water superficial velocity was increased, keeping constant $\mathrm{V}_{\mathrm{SG}}$, greater losses in the flow and greater accumulation of liquid in the bottom of the pipeline were reported.

5. Even though the slug flow existence was verified by using the literature models. It was found with CFD simulations that the pipe length used was insufficient to reproduce this flow pattern.

6. The CFD is suitably applicable in the behavior reproduction of the dispersed bubble and stratified smooth flow, obtaining reliable results with a deviation rate smaller than $20 \%$. 


\section{Nomenclature}

$\mathrm{V}_{S L}: \quad$ Liquid superficial velocities $[\mathrm{m} / \mathrm{s}]$

$\mathrm{V}_{S G}: \quad$ Gas superficial velocities $[\mathrm{m} / \mathrm{s}]$

$\phi_{\mathrm{B}}: \quad$ Bubble diameter $[\mathrm{mm}]$

$\Delta \mathrm{P}_{\text {TOTAL }}:$ Pressure total drop $[\mathrm{Pa}]$

Holdup: $\quad$ Liquid holdup

\section{References}

[1] Aziz K.and Petalas N., A mechanistic model for multiphase flow in pipes. SPE 9839. 1998.

[2] Wongwises, S., Khankaew, W. and Vetchsupakun, W., Prediction of liquid holdup in horizontal stratified two-phase flow. J. Sc. Tech.,Vol. 3, pp. 48$59,1998$.

[3] Shoham, O., Mechanistic modeling of gas-liquid: two-phase flow in pipes. SPE 97816. 2006.

[4] Taitel, Y. and Dukler, A., A model for predicting flow regime transitions in horizontal and near horizontal gas liquid flow. AIChE Journal, Vol. 22, pp. 47-55, 1976.

[5] Vásquez, F. Evaluation of a Biphasic Fluid Behavior Air-Water in a Horizontal Pipe through numeric tools of Computational Fluids Dynamics (CFD). Engineering thesis. University of Oriente. 2009.

[6] Beggs, H. and Brill, J., A study of two phase flow in pipes. J. Pet. Tech. Vol. 25, pp 607-617. 1973.

[7] Wallis, G.B., One-Dimensional Two-Phase Flow, McGraw-Hill Book Co.: New York. 1969.

[8] Lockhart, R. and Martinelli, R., Proposed correlation of data for isothermal two-phase, two-component flow in pipes. Chemical Engineering Science. Vol. 45, pp 39-48. 1949.

[9] Dukler, A. and Hubbard, G., A model for gas-liquid slug flow in horizontal and near horizontal tubes. Chemical Engineering Fundamentals. 1975.

[10] Vallée, C., Höhne, T. and Sühnel., Experiments and CFD simulation of stratified horizontal two-phase flow. International topical, project number 1501265.2005. 